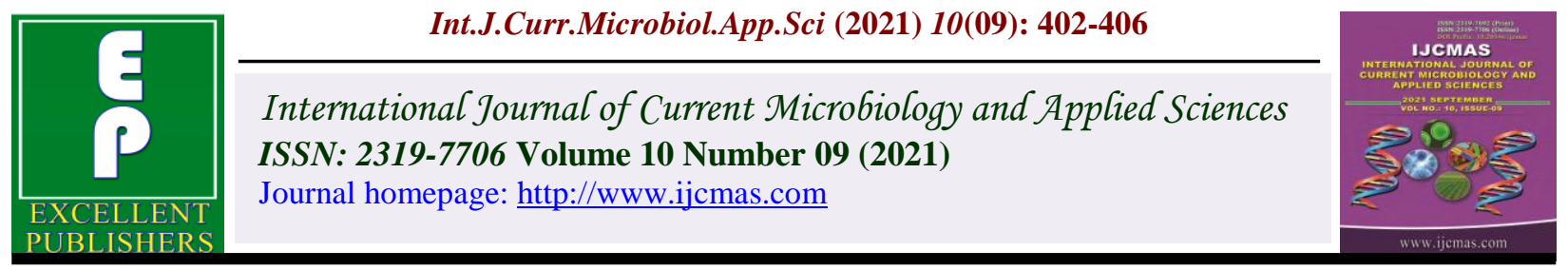

Case Study

https://doi.org/10.20546/ijcmas.2021.1009.046

\title{
Covid - 19 Associated Pulmonary Aspergillosis (CAPA) in Critically Ill Patients : A Case Report
}

\author{
Himadri Dutta ${ }^{1 *}$, Vandana Sinha ${ }^{2}$, Anup Jyoti Dutta ${ }^{2}$ and Pinku Sarma ${ }^{3}$ \\ ${ }^{1}$ Department of Microbiology, ${ }^{2}$ Department of Critical Care, ${ }^{3}$ Department of Radiology \\ and Imaging, Critical care, Ayursundra Superspecialty Hospital, Gorchuk, \\ Guwahati, Assam, India \\ *Corresponding author
}

A B S T R A C T

Keywords

Covid-19,

pulmonary aspergillosis,

CAPA, IAPA,

Co infection

Article Info

Accepted:

15 August 2021

Available Online:

10 September 2021
In the present study report three cases of covid-19 associated Pulmonary Aspergillosis from covid ICU of a private hospital. There is increased incidence of different co infections including fungal from severe cases of covid-19 requiring ICU care from different parts of the world. None of the patients in our report had any predisposing lung conditions and none of them were on long term steroids treatment. Out of the three cases, two cases clinically improved dramatically after initiation of antifungals. Second case deteriorated even before culture diagnosis was established and the patient died before antifungals could be started. So, possibility of a fungal infection should be always ruled out in all COVID-19 pneumonia cases without any clinical improvement after standard treatment and intensive care. Early diagnosis and treatment will help improve clinical outcomes in such cases.

\section{Introduction}

A novel corona virus later identified as SARS -COV-2, was first reported in Wuhan, China in late 2019 causing acute respiratory illness and gradually spread globally causing millions of death worldwide ${ }^{1,2}$. Many patients during the course of infection develop other associated infections which often remain undiagnosed which may lead to overall increase in mortality and morbidity ${ }^{3}$. With increasing ICU admissions (along with other infections), fungal infections has been reported increasingly in covid patients from China and from Nethelands. ${ }^{4,5,6}$.

We are here reporting 3 cases of Covid associated Pulmonary Aspergillosis (CAPA) from a multi-speciality private hospital covid ICU. 


\section{Case description}

\section{Case-1}

A 52 years old male uncontrolled type 2 diabetes mellitus presented to our hospital on 20/5/21 with severe breathing difficulty, preexisting Coronary artery disease, renal dysfunction and post covid complications. The patient had been diagnosed and treated for COVID-19 with steroids and other standard treatment for covid- $19^{7}$. The patient was on oxygen support from the day of admission. HRCT Thorax revealed post covid diffuse alveolar damage with cavitary lesions highly suggestive of Pulmonary Aspergillosis (Figure. 1). Chest Xray revealed post covid lung with cavitary lesions. Serum Galactomannan GM level was high (2.247) and was diagnosed as probable Invasive aspergillosis and treated with Inj. Caspofungin. Single sputum fungal culture and $\mathrm{KOH}$ were negative. The patient's respiratory functions improved significantly after antifungal therapy but kidney function deteriorated and hence airlifted to a higher centre.

\section{Case -2}

A 35 year old female without any significant past medical history was admitted to our covid ICU on 5/6/21 with severe respiratory distress. Patient was on Non-invasive ventilation (NIV) support initially along with standard treatment for covid- $19^{7}$ including steroids. HRCT Thorax on day 2 revealed bilateral covid pneumonia without any active cavitary lesion. On day 3 of admission, she was intubated with mechanical ventilator. Patient's inflammatory markers continued to rise. Patient was not a known diabetic but during her ICU stay blood sugar was raised (HBA1C -9) and insulin was started accordingly. Tracheal aspirate culture done by standard methods ${ }^{8}$ on 10/6/21 revealed pure growth of Aspergillus fumigatus after 48 hours (Figure.2). But unfortunately the Patient deteriorated before the antifungal treatment could be started and had cardiac arrest on 11/6/21.

\section{Case-3}

A 70 year old female diagnosed with covid19 , with previous history of hypertension and type 2 diabetes mellitus was admitted to our covid ICU on $8 / 6 / 21$. The patient was on oxygen support and her HRCT thorax on day 2 of admission was suggestive of progressive covid pneumonia. She was treated with standard treatment for covid- $19^{7}$ and steroids. However her follow up HRCT Thorax after 8 days revealed Aspergilloma involving lower lobe of the lung (Figure.3). Subsequently Serum galactomannan test (GM) was positive (3.12) and fungal culture (sputum) done by standard methods ${ }^{8}$ grew Aspergillus fumigatus. Accordingly covid associated aspergillosis was diagnosed and Antifungal medicines started. Patient improved clinically and discharged after 20 days of treatment.

We report here 3 cases of Covid associated Pulmonary Aspergillosis (CAPA) from critically ill covid-19 patients from our ICU. All the 3 patients were diabetic with uncontrolled sugar, on oxygen support and on steroid therapy. None of the patients had previous history of pulmonary tuberculosis/other chronic lung conditions. All the cases had radiological evidence of pulmonary Aspergillosis but none were positive for the European Organisation for Research and Treatment of Cancer (EORTC) and Mycoses Study Group Education and Research Consortium (MSGERC) ${ }^{9}$ host factor. The patient- 1 received corticosteroids before ICU admission and others received steroids during ICU stay; however, either the dose received was $<0.3 \mathrm{mg} / \mathrm{kg} / \mathrm{d}$ or the duration was $<3$ weeks. No immunosuppressive medication was given before CAPA diagnosis. 
Fig.1 HRCT thorax showing post covid diffuse alveolar damage with multiple thick walled cavitating lesion and intra cavitary components suggestive of Aspergillosis.

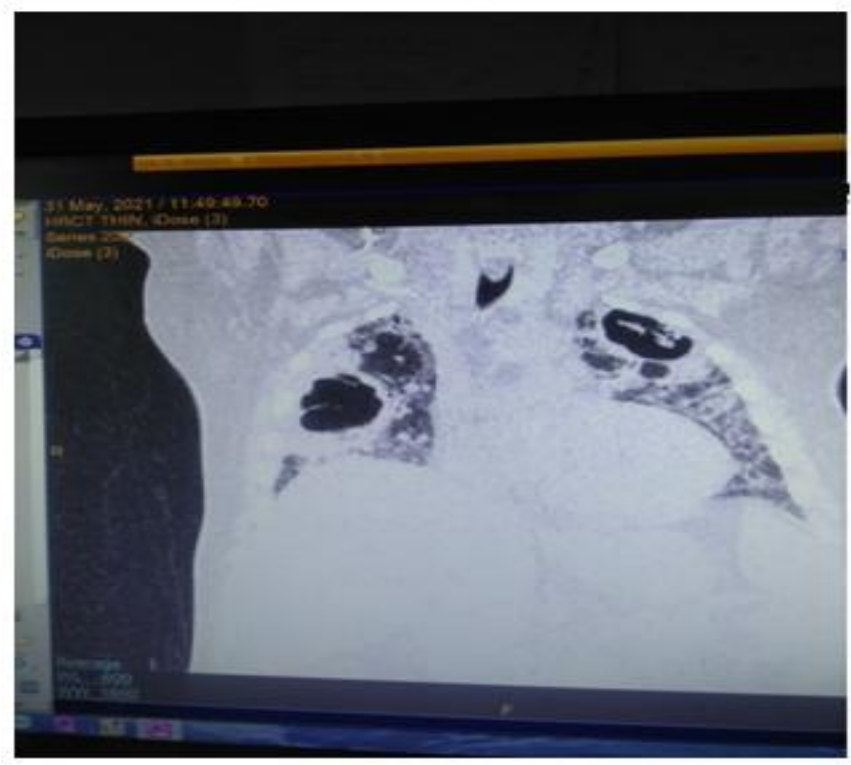

Fig.2 Lactophenol cotton blue mount (LPCB) showing hyaline septate hyphae with long conidiophore bearing vesicles with phialides covering upper two third of its surface

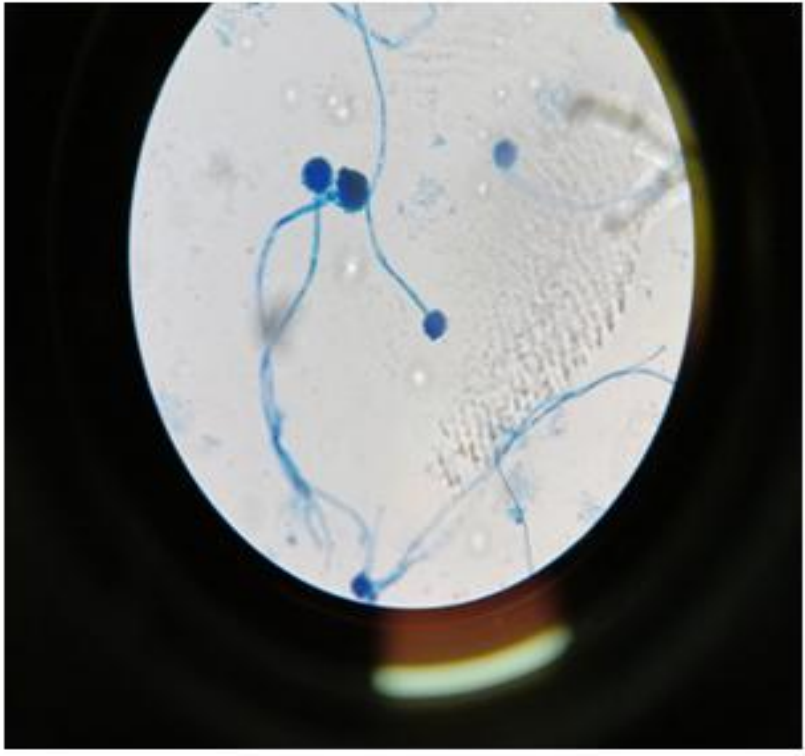

Fig.3 HRCT thorax showing absorption stage of covid-19 with left lower lobe thick walled cavitary lesion consistent with Aspergillosis.

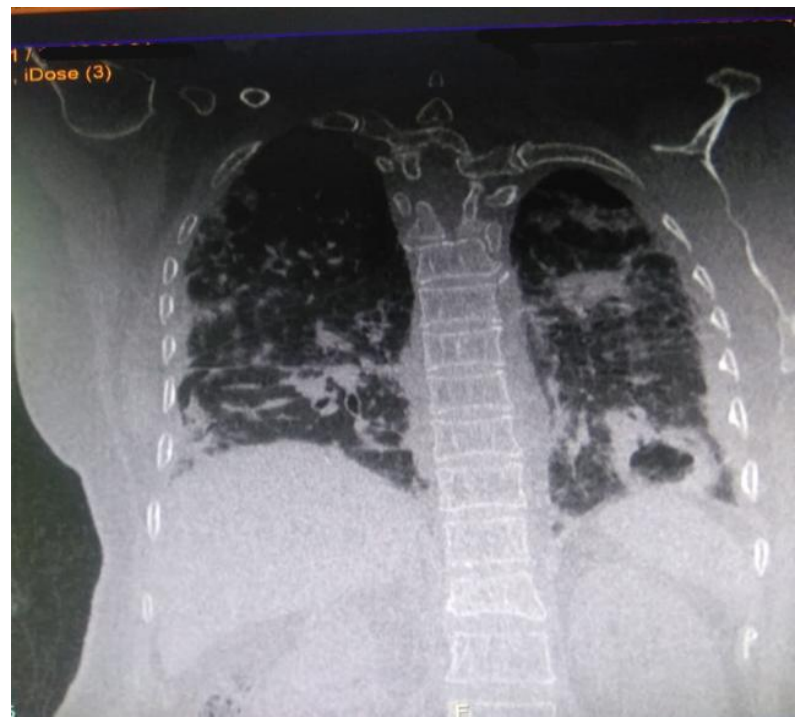

Except the case-2 who was on invasive mechanical ventilation for 4 days before the CAPA diagnosis, other 2 cases were on high flow oxygen therapy. The case- 1 had multiple comorbidities and his oxygen demand reduced after antifungals being started. Case- 3 also 
showed drastic clinical improvement after antifungal therapy initiation and was discharged after 20 days of intensive treatment. Case -1 and 3 were diagnosed as CAPA in immediate post covid period where as case -2 had CAPA after 5 days of ICU stay while being covid positive only and the fungal infection proved to be very fatal in this case.

As per latest consensus definition of invasive fungal disease ${ }^{9}$, Invasive aspergillosis is a fatal form of Aspergillosis usually affecting individuals with weakened immune system. There is increased concern that covid-19 patients are at more risk of developing various fungal infections including Pulmonary aspergillosis ${ }^{10,11}$.There are various reports of mucor infections among post covid patients from various regions of India, which is mainly attributed to prolonged steroid therapy and uncontrolled diabetes mellitus ${ }^{12}$.But other than mucor, other fungal infections like Aspergillosis are not much reported from COVID-19 patients undergoing treatment or as a post covid complication. Because of lack of availability of serological tests and microbiological culture, these infections remains undiagnosed.

Worldwide Influenza associated Pulmonary Aspergillosis (IAPA) has been reported from ICUs $^{13}$. There is a definite case definition for IAPA, but not for CAPA But if case definition of IAPA is followed considering COVID-19 pneumonia as the similar host factor as severe influenza, consideration of other factors like high GM level $>=0.5$ in serum and microbiological culture diagnosis, all the above 3 cases can be definitely considered as probable CAPA. The case -1 where single sputum culture was negative and unfortunately bronchoscopy could not be done, can be considered as possible CAPA with clinical deterioration. IAPA or CAPA both may remain undiagnosed because respiratory deterioration is considered to be caused mainly by bacterial coinfection and appropriate fungal diagnostics are not performed.

SARS-CoV-2 infection might be a risk factor for developing Pulmonary Aspergillosis (CAPA) and IPA. So Aspergillosis should be suspected in such patients with worsening clinical features with severe respiratory failure in ICU setting even in immunocompetent hosts. Early diagnostic work up with GM screening in serum and sputum/BAL, with fungal culture should be advised in all such patients.

\section{References}

1.Stop the Wuhan virus. Nature 2020;577:450. https://www.nature.com/articles/d4158 6-020-00153-x.

2.Munster VJ, Koopmans M, van Doremalen $\mathrm{N}$, van Riel D, de Wit E. A novel coronavirus emerging in China - key questions for impact assessment. $\mathrm{N}$ Engl J Med 2020;382:692-4.

3.Patti R. K., Dalsania N. R., Somal N. Subacute Aspergillosis "fungal balls" complicating COVID-19. J Investig Med High Impact Case Rep. Jan-Dec 2020;8 doi: $10.1177 / 2324709620966475$. [2324709620966475 ]

4.Yang X, Yu Y, Xu J, Shu H, Xia J, Liu H, et al., Clinical course and outcomes of critically ill patients with SARS-CoV2 pneumonia in Wuhan, China: a single-centered, retrospective, observational study. Lancet Respir Med. 2020.

5.Chen N, Zhou M, Dong X, Qu J, Gong F, Han Y, et al., Epidemiological and clinical characteristics of 99 cases of 2019 novel coronavirus pneumonia in Wuhan, China: a descriptive study. Lancet. 2020;395(10223):507-513.

6.Van Arkel A L E, Rijpstra T A, Belderbos H 
N A, van Wijngaarden P, Verweij P E, Bentvelsen R G. COVID-19 associated pulmonary aspergillosis. Am J Respir Crit Care Med. 2020.

7. Government of India. Ministry of Health \& Family Welfare. Clinical Management Protocol:

COVID-19[Internet] Available

from.https://www.mohfw.gov.in/pdf/U pdatedDetailedClinicalManagementPr otocolforCOVID19adultsdated240520 21.pdf.

8. Collee J G, Miles R S, Watt B. Tests for the identification of bacteria. In: Collee $\mathrm{J}$ G, Fraser A G, Marmion B P, Simmons A, editors. Mackie \& McCartney practical medical microbiology, $14^{\text {th }}$ ed. Edinburgh, UK: Churchill Livingstone; 1996. p. 13149.

9. Donnelly J P, Chen S C, Kauffman C A, Steinbach W J, Baddley J W, Verweij $\mathrm{P} \mathrm{E}$, et al., Revision and update of the consensus definitions of invasive fungal disease from the European Organisation for Research and Treatment of Cancer and the Mycoses Study Group Education and Research Consortium. Clin Infect Dis 20199 (ciz1008).

10. Zhu X., Ge Y., Wu T., Zhao K., Chen Y., Wu B. Co-infection with respiratory pathogens among COVID-2019 cases. Virus Res. 2020;285:198005.

11. Wang J., Yang Q., Zhang P., Sheng J., Zhou J., Qu T. Clinical characteristics of invasive pulmonary aspergillosis in patients with COVID-19 in Zhejiang, China: a retrospective case series. Crit Care. 2020;24:299.

12. Singh A K, Singh R, Joshi S R, Misra A (2021) Mucormycosis in COVID-19: a systematic review of cases reported worldwide and in India. Diab Metab Syndr: Clin Res Rev. 2021;15(4):102146.

13. Verweij P E, Rijnders B J A, Brüggemann R J M, Azoulay E, Bassetti M, Blot S, Calandra T, Clancy C J, Cornely O A, Chiller T, Depuydt P, Giacobbe D R, Janssen N A F, Kullberg B J, Lagrou K, Lass-Flörl C, Lewis R E, Liu P W, Lortholary $\mathrm{O}$, Maertens $\mathrm{J}$, MartinLoeches I, Nguyen M H, Patterson T F, Rogers T R, Schouten J A, Spriet I, Vanderbeke L, Wauters J, van de Veerdonk F L. Review of influenzaassociated pulmonary aspergillosis in ICU patients and proposal for a case definition: an expert opinion. Intensive Care Med. 2020;46:1524-1535. doi: 10.1007/s00134-020-06091-6.

\section{How to cite this article:}

Himadri Dutta, Vandana Sinha, Anup Jyoti Dutta and Pinku Sarma. 2021. Covid -19 Associated Pulmonary Aspergillosis (CAPA) in Critically Ill Patients : A Case Report. Int.J.Curr.Microbiol.App.Sci. 10(09): 402-406. doi: https://doi.org/10.20546/ijcmas.2021.1009.046 